\title{
The impact of rapid decrease of Aporia crataegi (Lepidoptera: Pieridae) population size on Wolbachia infection rate
}

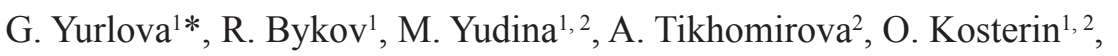
Yu. Ilinsky ${ }^{1,2}$

${ }^{1}$ Institute of Cytology and Genetics SB RAS, Novosibirsk, Russia

${ }^{2}$ Novosibirsk State University, Novosibirsk, Russia

*e-mail: yurlova@bionet.nsc.ru

Key words: Wolbachia, Aporia crataegi, population, mtDNA

Motivation and Aim: Wolbachia endosymbionts are widely distributed among insects. The high level of infection was observed in populations of black-veined white (Aporia crataegi) of Novosibirsk province in 2006. Rapid population decrease had occurred in 2007 due to late mid-May frosts. Here we examined Wolbachia infection rate in Novosibirsk A. crataegi population that restored its abundance in 2015-2016 seasons. Also we try to compare data of infection rates, Wolbachia and mtDNA diversity derived from different populations of black-veined white.

Methods and Algorithms: The collection includes $246 \mathrm{~A}$. crataegi specimens from Novosibirsk and Kemerovo provinces, Altai Republic, and Yakutia. Wolbachia infection status was determined by PCR with primers to coxA and 16SrRNA Wolbachia loci. The barcoding region of $C O I$ gene of both infected and uninfected specimens was sequenced. The phylogenetic tree $C O I$ gene was reconstructed in Mega 7 using all available sequences from BOLD database and our data.

Results: Wolbachia symbionts were not found in A. crataegi from Altai Republic, Novosibirsk and Kemerovo provinces, while several infected specimens were found in Yakutia. Their Wolbachia symbionts had coxA-6 allele. New alleles of A. crataegi mitochondrial gene ... were found.

Conclusion: Wolbachia infection rate in black-veined white of Novosibirsk province dramatically decreased after a severe drop of host population in 2007. No Wolbachia infection was detected at neighboring provinces as well. Also we found association of Wolbachia infection with a certain mtDNA allele of $A$. crataegi.

Acknowledgements: Supported by the RFBR (16-04-00980, 18-316-00099). 